\title{
The Proactive Security Toolkit and Applications
}

\author{
Boaz Barak Amir Herzberg Dalit Naor Eldad Shai \\ IBM Halfa Research Lab, Tel-Aviv Site \\ E-Commerce and Technologies Group \\ \{barak,amir,dalit,eldad@il.ibm.com\}
}

You can't cheat all people all of the time --(anonymous)

\begin{abstract}
Exıstıng security mechanıms focus on preventıon of penetrations. detection of a penetration and (manual) recovery tools Indeed attackers focus their penetration efforts on breaking into critical modules, and on avoiding detection of the attack. As a result, security tools and procedures may cause the attackers to lose control over a specific module (computer, account), since the attacker would rather lose control than risk detection of the attack. While controllıng the module, attacker may learn critical secret information or modify the module that make it much easier for the attacker to regain control over that module later Recent results in cryptography give some hope of improving this situation; they show that many fundamental security tasks can be achieved with proactive security Proactive security does not assume that there is any module completely secure against penetration Instead, we assume that at any given time perıod (day, week, . ), a sufficient number of the modules in the system are secure (not penetrated). The results obtaned so far include some of the most important cryptographic primitives such as signatures, secret sharıng, and secure communication However, there was no usable implementation, and several critical issues (for actual use) were not addressed

In this work we report on a practıcal toolkıt implementıng the key proactive security mechanisms The toolkit provides secure interfaces to make it easy for applications to recover from penetrations. The toolkit also addresses other critical implementation issues. such as the initialization of the proactive sccure system We describe the toolkit and discuss some of the potential applicatıons Some applications require mınımal enhancements to the existing implementations - e g. for secure logging (especially for intrusion detection), secure end-to-end communication and tımestamping Other applicatıons require more significant enhancements, mainly distribution over muitiple servers, examples are certıfication authority, key recovery, and secure file system or archive
\end{abstract}

Permission to make digital or hard copies of all or part of this work for personal or classroom use is granted without fee provided that copies are not made or distributed for protit or commercial advant -age and that copies bear this notice and the full citation on the first page To copy otherwise, to republish, to post on servers or to redistribute to lists, requires prior specific permission and/or a fee CCS '99 11/99 Singapore

(c) 1999 ACM $1-58113-148-8 / 99 / 0010 . \$ 5.00$

\section{Introduction}

Traditional security systems assume that one or more systems are always secure, $1 \mathrm{e}$ are never controlled by the attackers. The model of Proactive Security does not make this assumption. Instead, it considers cases where all components of the system may be broken-into and controlled by an attacker. with restrictions on the number of components broken-into during the same time period (day, week, ). Proactive security shows how to maintain the overall security of a system even under such conditions In particular it provides automated recovery of the security of individual components. avoidıng the use of expensive and inconvenient manual processes (except for some aggressive attacks, which cannot be prevented - but are definitely and clearly detected) The technique combines two well-known approaches to enhance the security of the system distributed (or threshold) cryptography. which ensures security as long as a threshold (say half) of the servers are not corrupted (see [12]); and periodic refresh (or update) of the sensitive data (e $g$ keys) held by the servers In short.

$$
\text { proactive }=\text { distributed }+ \text { refresh }
$$

This way, the proactive approach guarantees uninterrupted security as long as not 100 many servers are broken into at the same time Furthermore, it does not require identification when a system is broken into, or after the attacker loses control: instead, the system proactively invokes recovery procedures cvery so often, hoping to restore security to components over which the attacker lost control.

Proactive security is highly desırable in many realıstic settings, in particular:

- When a high level of security is required. together with fault tolerance (as redundancy improves fault tolerance but opens more points for attack)

- To ensure acceptable level of system security usıng weakly secure components such as most commercially available operatıng systems

(Examples of specıfic applications are given below.)

Recent results show that many fundamental cryptographic functionalıties may bc achieved even under the proactive security model - as long as most components are secure most of the time In particular, proactively secure protocols have been devised for the following problems

- Secret sharing $[21,16]$

- Discrete-log-based digital signatures [15], and in particular DSA [13] 
- Secure end-to-end communication [5]

- RSA [10,11.24], and in partıcular generatıon of the RSA shared key [3]

- Pseudo-random generation [6.8]

- Key distribution center [20]

This substantial set of known results in proactive security did not yet produce any practical security product or solution (In fact, there are only a few deployments of distributed security - the most well known may be the SET credit card standard's certıficate authority [7]: see also 'related works' below ) The creation of such a proactive solution is non-trivial, as the protocols are often quite complex and nontrivial to implement. Furthermore, the protocols are specified under some simplifying assumptions and do not address some needed elements, such as interfacing between the proactive service and the applications using it This paper reports on a toolkit. to be soon placed for public experimentation. to allow practical deployment of proactive security. The main new contributions are:

- A secure inttalization mechanism, with reasonable, practical requirements from the computer and operating system. Specifically, all we require is a secure boot process (which is a good idea anyway, against viruses - and easily done with signed code), and a per-machıne secret-private key pair, with the public key protected from modification (e g in ROM or write-once EEROM), and the seerel kcy in erasable memory (e $g$ disk). Previous results required storage of parameters specific to the particular application (such as the group's public key) in secure storage, which is not practical

- A set of application program interfaces (APIs) that allow the use of the toolkit to improve security, specifically provide security in spite of break-ins into computers, of existing applications, as well as the development of new applications which are proactive secure

The security of any proactive solution relies heavily upon its correct architeclure and integration with existing. non-proactive, operating system The design of our toolkit. which does not view the proactive model as series of protocols but. rather. as a security enhancement of the operatıng system which transforms it into a proactively secured system via the appropriate use of proactıve protocols, has not been defined nor implemented in the past. We show that it is possible to transform general applications which are required to remain secure for long periods of time to operate in a proactive environment. namely proactivizing applications. Specifically. we show how to appropriately use the proactive cryptographic functions as key primitıves in the proactivization process. To this end, we define an architecture for a proactive operating environment which serves as a platform on which standard applications can be proactıvized This operating environment consists of a network of servers which is set up once, which we call the proactive network Each server is instantiated at boot time by the operatıng system and is checked periodically. also by the operating system. Servers can recover data (both public and private data) from other servers in the proactive network, if such data is corrupted or lost Once the proactive network is set up, any applicatıon can run on the top of the network and request proactive services by the means of API. The feasibility of the proactive model and of the architecture presented hereby has been demonstrated by the Proactive Security Toolkit, which is a Java implementation described in this manuscript.

\subsection{Applications of the Proactive Security Toolkit}

There are three kinds of applications that may take advantage of the proactıve security toolkit to recover from penetrations.

Centralized applications - a 'traditional' application running on one server only. The application uses a proactively secure service provided by the toolkit For some applications and services, this could provide significant advantage - at minımal change to existıng applications Some typical applications are:

- Secure logging: each client application may add entries (cvents) to the log, however none of them can modify or erase the log. This could be of great value in improving intrusion detection tools, as intruders often try to erase traces in $\log$ files.

- Secure end-to-end communication: the proactive toolkit can provide the applications with freshly generated and certified public keys perıdically. This could be integrated with tunneling mechanisms such as secure IP or SSL

- Timestamping: the toolkut could be used to sign a document (or its hash) and current time, to prove that the document existed at this time

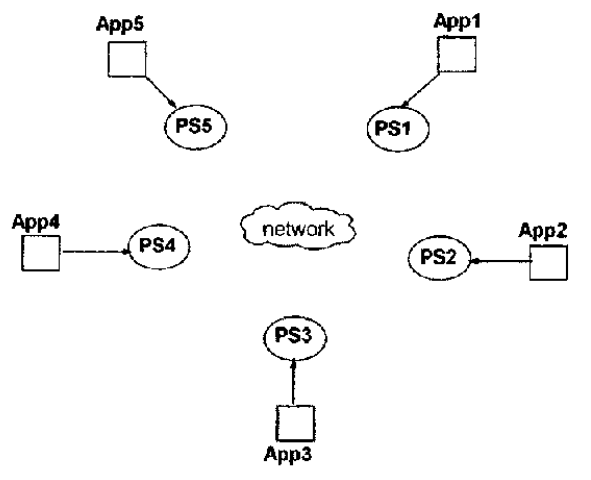

Figure 1: Distributed Application of the Toolkit

Distributed applications (Figure 1 ) - the application runs simultaneously on all machınes (App_1, , App_n) and requests services through all machines. Each App_i interacts directly with its own proactive server (PS_i). A typical application is a certificate authority, or in general any workflow application requiring secure (multi-person) digital signatures Another application is key recovery (escrow agents).

Proactive applications - the applıcalion runs in a distributed configuration but, in addition, goes through periodıcal refreshes by utılızing the proactıve toolkit services. This is required when the application security or efficiency requirements cannot be met by the services of the toolkit. Examples include multiparty protocols such as voting and trading, database, operating system. and access control mechanisms An especially interesting application is a Secure Commerce Server - such server can not lie within the firewall although it handles confidential data and 
matters (such as access control, certificates etc) It is therefore natural to proactively distribute the server among a number of (independent, and possibly not even mutually trusted) hosts and locations, thus achieving increased trust in the server.

\subsection{Related Systems}

A number of distributed systems related to the proactive model have been designed and implemented. The Intrusion Tolerance via Threshold Cryptography (ITTC) project [17,19,29] desıgns and builds tools and an infrastructure that are based on Threshold Cryptography, and use an intrusion tolerant Certification Authority and an intrusion tolerant web server to demonstrate these tools. The technical approach is based upon a distributed RSA key. such that $k$ "share servers" generate a private RS $\Lambda$ key that is shared among them from the moment of creation, and any $t$ of the share serves can be used to apply the key Another related system is the Omega Key Management Service system [27]. designed and developed at Bell-Labs. The $\Omega$ system is a distributed public key management system It employs threshold techniques which can tolerate a number of server failures (via the implementation of the Rampart toolkit [25]), but not recovering (proactive) techniques. The $e^{-V a u l t}$ (electronic-Vault) project [18] of IBM is an implementation of a distributed data repository It employs RSA-based shared signatures as the basic cryptographıc function.

There are a few implementation efforts of proactive algorithms. Specifically, the Network Randomization Protocol (NRP) of [8], which provides a proactive pseudo-random generator, has been implemented at IBM. It also provides a simple API for client applications to get pseudo-random values from the servers Another effort, the implementation of proactive threshold key protocols, has been reported in [14].

\subsection{Organization of the paper}

This paper is organized as follows In Section 2 we describe the model as well as an overview of related work. Section 3 discusses the basic architecture of the proactive server Sections 4 presents the protocol that intializes the network of servers and handles refresh/recovery of a server The API module, which provides mechanisms for proactivization of applications, is fully described in Section 5 Java-related and other implementation issues, user-interface are the focus of Section 6 .

\section{Overview of the Proactive Model and Algorithms}

\subsection{Model}

The proacuve model assumes a set of $n$ servers, $\{P l, P 2, \quad P n\}$, that are interconnected by a complete point-to-point communication channels Time is divided into periods (like days, weeks,. ) which are determıned by some global clock. An adversary may (temporarily) attack up to $t$ of the $n$ servers at any given tıme period - but at different time periods, different sets of $t$ servers can be attacked. As a result, all servers engage in a refreshment stage at the beginning of each time period, so that any server which has been attacked during past periods may automatıcally recover from possible undetected break-1ns
Corruption is assumed to be either static (for example, disconnect a server from the rest of the network, eavesdrop, read secret data) or active/malicious (for example, devate from the protocol. corrupt local data etc) Therefore, after the attacker loses control over a server, the attacker may still know secret information of that server (e g passwords or secret keys) Furthermore, before losing control, the attacker may have corrupted (modified) some of the server's data (e.g. public keys of certificate authorities). The refreshment stage deals with both aspects, $1 \mathrm{e}$ recovers any corrupted data and invalıdates any old secret data (by choosing new secrets or splitting global secrets into a new set of shares) This brings the server back to a running stage, and guarantees that any information that was gathered by the adversary becomes worthless after recovery

The fact that we limit the attacker to $t$ corruptions, out of $n$ servers, is similar to the distributed (or threshold) security model used in many works in distributed computıng and cryptography. However, in the proactive security model we allow the attacker to corrupt every server - as long as it does not corrupt more than $t$ servers at the same period We say that adversary in the proactive model is mobile, namely attacked components may be released at some point (due to some security measure or other change in the system or the adversary causing loss of control, often as a result of an attempt by the adversary to avold detection of the attack) Furthermore, in contrast to other approaches, proactively secure systems do not wait untıl a break-in is detected Instead. a proactively secure system invokes the refreshment protocol periodically (and proactively) in order to maintain uninterrupted security, or force detection For more discussion on the motivation behind this model, see $[4,5,6,16]$

Some attacks on the system cannot be prevented The 'classical' example is if the attacker is breakıng into a server, thereby finding all its secret keys; it then pretends to be that server while keeping this server disconnected from the other servers (when the attacker lost control over that server) However, in such cases we will be able to detect the attack, and raise an alert - inform the operator about the attack Operators w1ll normally respond to such an alert by invoking special cmergency security resources and procedures, which are very likely to remove the attacker - and possibly catch her as well Therefore, it is highly unlikely that (smart) attackers will use such 'visible’ attacks

The proactive security model assumes that even durıng attack, some specific data cannot be corrupled The obvious example for data that we must assume cannot be corrupted is the program itself: if it could be changed, recovery is clearly impossible Clearly, the program is not any different than any constant value used by the program; we will therefore assume that each computer comes with a read only memory which we can specify its contents Specifically we assume that each computer comes with such a read only memory containing a fixed public key, and the corresponding secret key is known only at intialization - for a more detalled discussion, see Sectıon 3.2. This assumption is not too difficult to implement in practice

\subsection{Toolkit's Functionalities and Algorithms}


Our toolkıt maintains two basic proactive functionalitıes for the entire lifetime of the system, as long as there are 'enough' working components in the system:

1 Proactively secure end-to-end communication (authenticated and encrypted) among all the nodes of the proactive networks, that is, new communication keys are agreed upon at the beginning of each period This functionality is achieved using the protocols of [5]

2 A distributed signature key that is generated at initiation of the proactive environment, shared among all servers of the network and proactively maintained so that private shares are refreshed periodically without changing the signature public key This internal signature key is often used for 'group certification' purposes and. for example, is mandatory for the implementation of the proactive cnd-lo-end communication Our toolkit implements a DSS distributed key using the algorithms of $[15,13]$. but in principal it is also possible to use a distributed RSA key, based on the signature algorithm of [24.11] with the hey generation algorithm of [3].

The implementation of these functionalities are based on a number of algorithms which, for completencss, are briefly outlined in the Appendix of the paper"s ful version (http / w3 research telaviv ibm com/proactıve/Papers/Toolkıt/proa ctive-paper ps)

\section{The Proactive Toolkit Architecture}

Recall that the proactive operating environment serves as a platform on which standard applications can be proactivized in this section we define the basic architecture and functional components for such environment.

The proactive operating environment consists of a network of servers which is set up once - this network is referred to as the Proactive Network Fach node in the network runs a proactive server (PServer), whose basic architecture is depicted in Figure 2 A Pserver communicates with other Pservers via the proactive network. and provides proactive services to applications by the means of API A server is instantiated at boot time and checked periodically by the operatıng system Current implementatıon does not support dynamic resizing of the network

The internal design of a Pserver is composed of the following modules:

- Library of Proactive Utılities

- Library of Proactive Protocols

- The API Module (section 5)

- The Controller and Communication modules

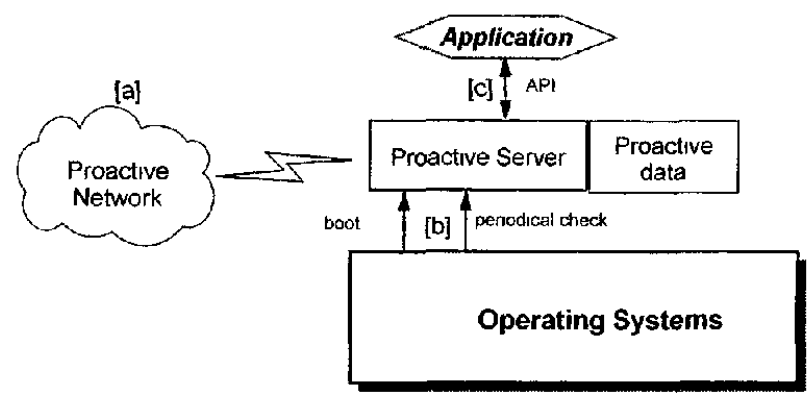

Figure 2: Architecture

\subsection{The Pserver Data}

The Pserver, as any other program, maintains some key internal data However, the mairtenance of this data raises a few algorithmic problems, as the server must be able to refresh and recover itself periodically, and this includes recovering its data or at least verifyıng that it has not been corrupted The server's data is one of three types

1 ROM data - this "write once" data is assumed to be immutable so that any attack on the system can not tamper with it, however an adversary may learn it It is used for bootstrapping purposes as otherwise a recovering server could not bring itself to a secured state. Our design. as detailed in Section 4, attempts to mınimize the amount of data that must be stored in the ROM in order to safely boot the server: in particular it shows that it suffices to store one public key (in our specific implementation, the server's port number as well) in the ROM for the Pserver to be completely recoverable

2 Public data Parts of this data are common to all servers. but other parts are specific to the particular Pserver, yet its exposure to the entire proactive network does not interfere with the security of a PServer. Since this data is necessary for the proper operation of any server and thus must be recoverable, it is duplicated the data among all servers so that during recovery it can be reconstructed if needed with the assistance of the proactive network The detarls of this process are described in Section 4. The public data may be extended during the lifetime of the system. for example by gencrating new long-lived secrets (the common fields of these long-lived secrets is added to the public information)

3. Private data, specific to a particular server One such example is the server"s share of a distributed key This data is typically not recovered, but instead is refreshed It also requires the ability to be completely erased from the system without lcaving any traces, which is a property that needs to be supported by the operating system.

\section{The Proactive Toolkit Protocols}

Our suggested design for a proactuve operating environment must maintain proactively secure communication among the servers as well as a proactıve internal signature key for the entıre lıfetime of 
the system For that these two protocols must be inttiated and undergo refresh at every period, where a refresh may actually involve recovery at some server if it had detected that some of its data was corrupted or lost We follow [5] for the design of the integrated proactive protocols of signatures and secure communication, and $[13,15]$ for the specific proactive signature mechanism However, [5] requires every server to keep in read-only memory (ROM) a copy of the verification key Vcert of proactive system (whose corresponding secret key Scert is shared between all the proactive servers, and these shares $S_{\text {cert }}(t)$ are refreshed at every period $t$ ). This assumption is not very practical, as the proactive system's key Vert is not available when the computer is manufactured and sold, but only much later - when it is integrated into a specific proactive environment We show how to provide the requirements of [5] while requiring only that each computer comes with pre-installed, machine-unique pair of secret key $S_{\text {เart }}$ (on erasable disk) and public key $\nu_{\text {sorr }}$ (on ROM)

Another pracucal aspect which we had to deal with is that the proactive server needs some constants configuration information such as IP addresses of other servers, cryptographic parameters used in the cryptographic algorithms, and so on We denote this set of these (public) constants by $C$. Our protocols include mechanusms to recover $\mathrm{C}$ periodically (if the adversary corrupted $\mathrm{C}$ when breaking into the server at the previous period). Let $M l=\left[S_{\mathrm{t} i m}\left(V_{\mathrm{cor},}, C\right)\right]$ be the signature of server $i$ on $\left(V_{\text {cert }}, C\right)$ using its initıal key $S_{\text {⿶(rm }}$ We denote by $M$ the concatenation of all $M i$ s. that is $M=\left(M_{1}, M_{2}, \quad M_{n}\right)$ Hence, $M$ is the Invartant Information of the system

We begin by briefly reviewing the periodical refresh protocol of [5], which assumes the avallabulity of an unmodified Vcert at every proactive server We then describe the Vcert-recover protocol, with a periodical-recover module which recovers $/$ cert at the beginning of every refresh period (before using [5]), and an intialization module that uses $\left(S_{s t a r}^{\prime}, V^{\prime}{ }_{s m}\right)$

\subsection{The Refresh Protocol of [5] for Period t}

The goal of this sub-protocol, detailed in Table 1, is to refresh the communication/authentication keys as well as the shares of all long-lived keys, including Scert. A server which partıcıpates in this protocol may be "operational", in which case it has a valid parr of keys $\left(S_{l}(t-1), V_{l}(t-l)\right),\left(E_{l}(t-1), D_{l}(t-1)\right)$ and valid shares of the long-lived secrets (including $S_{c c r}^{\prime}(t-l)$ ) from period $t-1$

Alternatively, a server can be "recovering" so that all of the above information is missing (or corrupted), even in this case we can assume that server posses Vcert and the constants $C$ (to be ensured by the $V$ cert-recover protocol)

\section{Remarks:}

1 Step 3 of the standard key refresh allows the option of sendıng $K l$ in the clear. since a recovering server has no valıd $S i(t-1)$ at this point. Hence, the following judgment should be made:

[1] If more than one (but different) authenticated messages arrive from server $i$, discard all of them

[ii] if one authenticated message arrives, but few others in the clear, accept authenticated

[1ii] If more than one (but different) messages arrive in the clear, discard all of them

2 Recovering servers do not take part in the Joint-signature generatıon.

3. When verifying the signature Scert(KeyTable) server $I$ must venfy that Key Table contains $K_{l}$ (this serves as a "random challenge" to avoid replay of signature). If server $t$ discovers that $K_{l}$ is not part of the signed KeyTable, then raise an alert for a detected attack

4. Use the secret recovery and refresh algorithms for proactıve secret sharing, as described in [16]

\author{
Perform a standard key refresh for period $t$ : \\ 1. Generate $(S i(t), V u(t))$ \\ 2 Gencrate $\left(E_{l}(t), D_{l}(t)\right)$, Sign with $S_{l}(t)$ \\ 3. Broadcast $K_{l}=\left(V_{l}(t), S_{l}(t)\left(\left(E_{l}(t)\right)\right)\right.$ (using $S_{l}(t-1)$ when avallable) ['] \\ 4 Generate a distributed signature Scert $($ Key Table $)$ where KeyTable $=[\mathrm{Kl}, \quad, \mathrm{Kn}]\left[{ }^{2}\right]$ \\ 5. Verify the signature Scert(KeyTable) $\left[^{3}\right]$ \\ (All channels are now authenticated/encrypted with refreshed keys of period $t$.) \\ Refresh long-lived data $\left(S_{c c r t}\right)$ : \\ 1 Perform an agreement protocol on the data that needs to be refreshed/recovered \\ 2 For any long lived secret $S\left[{ }^{4}\right]$ \\ (i) Reconstruct the missing shares of the secret $S$ for the recovering servers \\ (ii) Engage in a standard Refresh (S) protocol
}

Table 1: Refresh Protocol for period t 


\section{PushM algorithm of server $i$}

Read $V_{\text {stom }}$ from ROM and check validity of the stgnature [Scert $\left.(M), M\right]$ stored in a file:

(i) Extract $M l$ from $M$ and check validity of signature of $M l$ with $V_{\text {vart }}$

(ii) If signature on $M l$ is valid. obtain $V c e r t$ from $M l$ and valıdate signature $S$ cert( $M$ )

If signature on $M$ is valid - server $i$ is operational

- Send $M$ to all other scrvers

Otherwise ( $M$ is not valid or file does not exist) - server $i$ is recovering

- Wart until a verffiable copy of $[S c e r t(M), M]$ arnves from other servers (otherwise raise ALERT - recovery has farled)

Table 2: The Vcert-recover Protocol - periodical-recover module

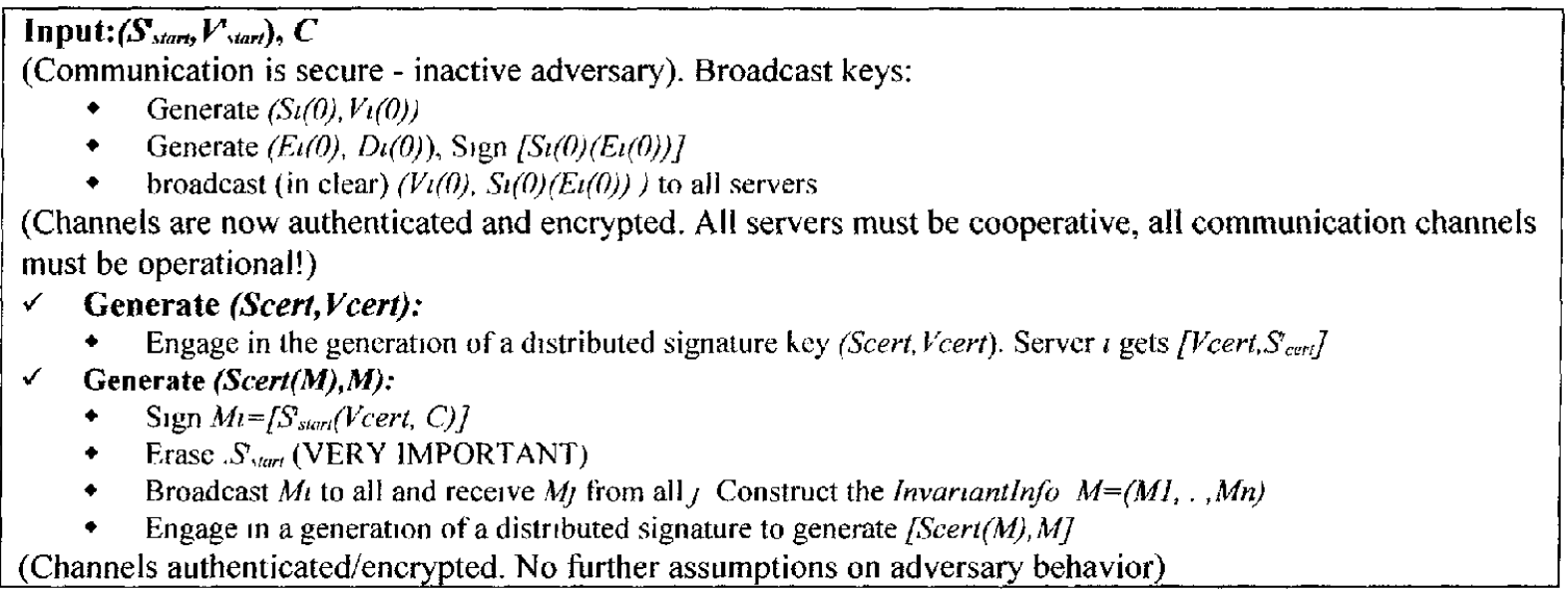

Table 3: Initialization Protocol of server $i$

\subsection{The Vcert-recover Protocol - periodical-recover module}

The penodical-recover module, detailed in Table 2 , is invoked at the very beginning of very refresh period. and re-generates $V$ cert and the constants $C$ for any server which lost this data. As a result, it brings a recovering server to a state from which it can participate in the Refresh protocol described above We assume that any operational server has a valid copy of a signature on $M$, the Invariant Information of the system. signed by the distributed signature key Scert - an assumption that is justufies by the initialization module described next

Essentially, this protocol allows any recovering server to gather $M$, the Invariant Information of the system. from other operational servers as long as there are enough of them Note that $M$ needs to be "pushed" around the system since a recovering server may not know who its partners are (recall that $C$, the program constants, contauns information such as IP addresses). The protocol is executed by all servers. and by the end of it a server detects whether it is "operational" or "recoverng"

\subsection{The Vcert-recover Protocol - initialization module}

This protocol is exccuted at the setup of the system lts goal is to bring the servers to a state from which they can safely perform the periodical-recover module at every Refresh stage and achieve proper operation of the system The protocol does the following it first generates the initıal set of authentication/encryptıon keys of the system, it generates the distributed signature key ( $/$ cert, Scert) and finally produces a jount signature $[\operatorname{Scert}(M), M]$ on the Invariant information to help recovering servers bootstrap their data in the future The input to this protocol is $C$, the program's constants, and $\left(S_{\text {tart }} V_{\text {varr }}\right)$ where $V_{\text {stat }}$, the public part of this key, is also written in the ROM. Table 3 summarizes the detals of the Intialization protocol

\section{The Application Program Interface (API) Module}

This section describes the interface between the proactive toolkıt and the applications using it A centralized application runs on the same computer running the proactive server, distributed or proactive applications (Figure 1) run one instance of the application on each of the proactive servers The goals of the API are to provide secure communication between the application and the server We will assume that the operating system is providing basic security services which allow server and client to restrict communication to the same computer, and to separate between two applications There is one element of security that we must add in the API, which is, how to identify multiple instances of the same application runnung on the different servers - this will be done with a secure registratıon mechanısm. The API's categones are. 
1 Registration API's (must be used first. to get a handle to be used for other API calls)

2 Data Storage API's

3. Communication APl's

4 Service API's

\subsection{Registration API's}

Since a Pserver can possibly service many clients, it is necessary to provide an authentication mechanism for requests, by which a request is uniquely associated with the client application it originated from (the application's "name") For example, if an application by the name of "VerıSign_CertificateAuthority" is registered at the proactive server, asks for a generation of a proactive signature key and then repeatedly asks to proactively sign messages by this key. then the server needs to authenticate these requests The registration mechanism is designed to address exactly this problem Registration will provide the application with a handle. which it will append to every subsequent request Different registration mechanisms are needcd for different client configurations (centralızed vs distributed/proactive)

Centralized Application - this is the straightforward registration. designed to support requests that are initiated by a single client The client sends

Registration_Req(Name, UniquelD)

where UniquelD is some random idenufier of the application, the server responds with a handle

The server needs to assign a quota on the number of services a spectfic client can request

Distributed Application - The group of Pservers need to identıfy clients with the same name running on different machmes In particular, a request will be serviced by the proactive servers only if a majority of authenticated clients have intrated (or approved) this request We suggest two options

1 Password based: Each client must register at its Pserver with the same (name,pswd)

Client_l sends Registration_Req(name, pswd) to Pserver_ $t$ Pserver $t$. returns a handle apt handle $t$ to its client A request is serviced only if requested by a majority of servers For that, if $P_{\text {server }} t$ gets a request Request_protocol(apl handle_t, protocol) from Client_t. then Pserver $l$ sends (hash(pswd), name. protocol) to all If at some Psierver, no such request has arrived, it asks for approval from its cllent $J$ (after checkıng hash(pswd))

2 Certificate based Assume a Certıficate Authority common to all clients Prior to registration at the Pserver, each Client_1 gets a one-time certificate

Certificate_l $=$ Cert_CA(Client_PubKey, name,, , server_ld, timej

This certificate is used to convince Pserver_t that the registration request indeed comes from a valid client which is the 1_th component of some application named name (that is, If the same name is used at various clients, then they are instantiations of the same application) Of course, the application must also prove ownership of the secret key corresponding to the public key in the certificate (by signing time or a challenge from the proactuve scrver) This protocol is depicted in Figure 3

The password solution is much weaker since if one finds out the password it can "forge" a clıent at all other machınes at once, whereas a different certificate (and secret key) is needed at each machine.

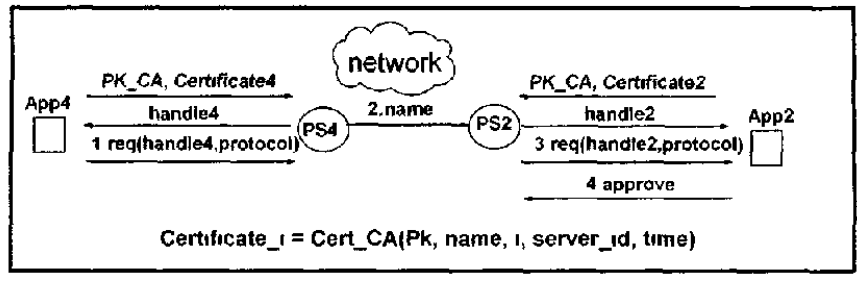

Figure 3: Certificate-based registration for distributed applications

\subsection{Data Storage API's}

These APIs provide secure store and recovery functionalities for the application data which are all based on the various Secret Sharıng algorithmic techniques The API supports the following functions

StoreData (DatalD. DataValue, data_type)

Retrieve (DatalD)

Write (DatalD, newlalue)

where DatalD uniquely identifies the data entry, and data_tvpe indicates what securtty optrons/requirements apply to this data Below we categorize the various types of data, specifically, data can be either read/write or write-once memory, can be public or private, distributed or local. Table 4 summarizes these types

- Public (non-secret) data can be requested to be stored and retrieved either as a write-once data or with read/write privileges. The latter case makes no sense in a local (central) application, since then it could be erased by a single corrupted server while under attack Hence, in order to change the secret value (namely, perform a write operation), a quorum of the Pscrvers must request the operation

- Secret write-once data can either be "single writer/reader", hence it is private to its owner but is distributed (vta secret sharıng) among servers for security and tolerance, or "sıngle writer/dıstributed reader" data whıch has already been distributed among the servers elsewhere and can be $\mathrm{read} / \mathrm{reconstructed} \mathrm{only} \mathrm{if} \mathrm{a} \mathrm{quorum} \mathrm{of} \mathrm{the} \mathrm{servers} \mathrm{request} \mathrm{to}$ read it. Note that the system automatically provides refresh and recovery of shares for this type of data.

- Secret read/write is "distributed writer/distributed reader". It requires a quorum of the servers to change its value, hence it is applicable only in the distributed scenario

An additional interesting secure storage service is Store Untul (DatalD, Data Value, Date) This is a special interesting service that is derived from the "single writer/distributed reader" or "distributed writer/distributed reader" variant The data is kept secret unt1l the specified date

$\left.\begin{array}{|l|l|l|l|}\hline \text { Read/Write Data } & \text { must accept request for } & \begin{array}{l}\text { Same as write-once, but the } \\ \text { secret value may be changed }\end{array}\end{array}\right\}$ N.A.




\begin{tabular}{|l|l|l|l|} 
& $\begin{array}{l}\text { store/retrieve from a quorum } \\
\text { of servers }\end{array}$ & $\begin{array}{l}\text { if requested by a quorum of } \\
\text { servers }\end{array}$ & \\
\hline Write Once Data & $\begin{array}{l}\text { accepts request for } \\
\text { store/retrieve from one } \\
\text { server }\end{array}$ & $\begin{array}{l}\text { "single writer/distributed } \\
\text { reader". } \\
\text { Receive secret shares that } \\
\text { are computed elsewhere (by } \\
\text { the app). } \\
\text { Perform periodical refresh } \\
\text { and share recovery when } \\
\text { needed }\end{array}$ & $\begin{array}{l}\text { "single writer/reader" } \\
\text { Only owner may } \\
\text { reconstruct. } \\
\text { Can not be modified } \\
\text { Perform periodical refresh } \\
\text { and share recovery when } \\
\text { needed. }\end{array}$ \\
\hline & Public (not secret) & distributed & Private \\
\hline
\end{tabular}

Table 4: Data Storgae API

\subsection{Service API's}

The following services are readily avallable from the proactive network.

- GenerateDSSKey (params) - Pservers engage in the generation of a distributed DSS key

- GenerateDSASignature (message, DSSKey) - Pservers engage in the generation of a distributed signature, using the algorthm of [13].

- GetTime - returns a vector with the local time at each proactive server.

- GetRandom() - every Pserver generates and sends a random number, return XOR on them.

- GenerateJointSecret) - engage in the protocol that generates a jount secret. Secret value can be etther specified or a random value.

\subsection{Communication API's}

The proactive toolkit can supply means for proactively secure communication between two nodes, etther by supplyıng proactıve keys. or by supplying proactive communication-links

Proactive Keys - Communication applications often require generation of session keys, and these heys need to be refreshed from time to time Such refreshed keys can be generated by the proactive toolkit and supplied to the application, together with certificate (signed by the proactive key of the Pservers) which certffies the public key of penod $t\left(\mathrm{~K}_{\mathrm{t}} \mathrm{t}\right)$

Proactively secure communication links - Since the Pservers already maintain proactively secured communication among themselves. this mechanism can be provided as a service to an external application To achieve that, an application first needs to register as a client on both servers (using the same name) and then use the send/recerve API's between the client and the Pservers.

\section{Implementation}

The Proactive Security Toolktt has been prototyped in Java $11 \mathrm{~A}$ beta version of the toolkıt will be available by YE 99 for public experimentation, and a running demo is in our web stte

Performance: The toolkit's current implementation serves mainly as a feasibility study for the proactive model As such, it does not consuder performance as its primary goal. and indeed was developed in Java for fast prototyping rather than to achieve good performance Moreover, since proactive algorithms typically have a bursty communication pattern, the communication bottleneck constitutes the performance barrier Therefore, time performance is basically a function of two parameters $d$ - the maximum delay on a point-to-point communication that the system expects. and $t$ the maximum number of bad servers. $t$ directly affects the complexity of the step which broadcasts a message to all servers, a fundamental step in all protocols proactive

Rough estimations show that with current implementation the toolkit's heavy tasks are performed off-line (during refresh) and require the order of 10 minutes for a complete periodical refresh ( for $n=5$ ). An important on-line task is the generation of a signature. This task requires the order of 4 broadcast steps, where a single broadcast takes about $t d$ time

\subsection{Java Related Implementation Issues}

The proactive environment architecture and its algonthms constitute quite a complex system to implement and test As such, the Java language was a natural choice for implementation sunce it provides a fast and simple prototyping environment. Moreover, its portability across platforms was an important feature. since different nodes in the proactive network may have to run the toolkıt on entirely different platforms (for example, our demo runs on a network of five nodes, some of which are UNIX based and others are Windows based). Yet. besides its poor performance, this choicc of programming lagnguage had a number of implications

1. Erasing information from memory, which is an absolutely nccessity for the correct implementation of secrets refresh, is an issue in all environments (due to virtual memory) and, in particular, in a garbage collected environment lıke Java, since garbage collectors typically copy memory as part of the collection proccess.

2 The proactive model assumes that after the adversary has lost control on a machine the code of the proactive server program is valid, so it is either protected by some tampered-proof memory device or can be safely loaded by the operating system (see the API section). The code for a Java program includes the code for the IVM (Java Virtual Machıne), as well as the byte-code of all classes loaded (dynamically) by the machine in the course of its execution Therefore, satısfying the code-valıdation assumption for Java programs may require assistance nol only from the Operatıng System, but also from the JVM, possibly by using 
mechanısms like signed classes or by writıng a customized class loader.

3 We were able to use some of the more advanced features of Java to simplify both the protocols and communication (by using serialization), and the API (by using dynamic class loading and reflection). All protocols and messages are implemented as subclasses of an abstract superclass. In this way all protocols are treated in a uniform way, which simplifies both the dispatch of messages to protocols and the addition of new protocols. In addition, we didn't have to define 'protocol messages' in a strict, well structured, way and parse them. Instead, all messages are sent as serialization of some object.

\subsection{Signing an agreed-upon object}

The following implementation issue. not necessarily exclusive to Java , ss relevant in order to jointly sign and valıdate an agreed-upon object An object is signed by first converting it to a number. but a conventional conversion may not guarantee that identical objects will be converted identically. For example, if two identical sets are implemented via linked lists then the representations may be different due to distinct orders within the lists.

In our mplementation, it is often desirable that all "good" servers sign an identical object which they all posses For that, the following protocol has been used:

- Each server broadcasts a byte array which is a serialızation of the object

- A server accepts the byte array which is the serialızation of an identical object to its own, and comes from the lowest indexed server

\subsection{API Implementation}

Using the Java language enabled us to implement the API between the server and its clients in a convenient and simple way, simılar to the API for writing applets. To write a proactive application the client must write a class which is a subclass of the class ProactiveApplet which is part of the toolkit This superclass provides its subclass with methods to request services from the server, send messages to clients running on the other machines, and load new classes to the proactive server. In addition, this class defines abstract methods which the subclass must implement and which the server uses to notify the client about the status of request and about incoming messages from other clients. The class also defines an interface which the client implements to allow its data to be saved and restored from the server In short, a large part of the API specifications outlined in Section 5 is already provided, either as methods, abstract methods or interfaces, of the ProactiveApplet superclass

As a result, cxecutıng a "client" application is essentially reduced to loading a class which is a subclass of ProactiveApplet into the JVM executing the Pserver class, and the issue of registering the client application is now reduced to an authorization/policy mechanism to allow the loading of this class. To this end, we suggest that the code for some predefined list of classes is part of the initual constants $C$ of the server, and, as mentioned above, these classes can invoke methods to load new classes (in the same package or subpackages - to avoid namespace conflicts). A class is loaded at the next periodic refresh if a majority of the servers received a request from a client to load it The code for all loaded classes is also validated at each refresh. Therefore, the server trusts classes that have been loaded to it, and the classes that are loaded initially are responsible for implementing the policy of which new client classes to load

One natural policy that can be employed is via "signed code" mechanisms provided by the Java language We intend to supply standard initial classes which provide a GUI interface to request loading of new classes, and which will only load classes that are signed by a predetermined certificate authority However. different initial classes can implement different policies such as a "class that will delay requests for loading new classes for a week, while notifyıng managers and requesting their authorizatıon". Some initial classes may decided not to load classes at all, but listen for requests through a TCP socket and decide whether to accept them on a per request basis (such a class can act as a proxy for a non-Java or a non-local client application)

Since there are no inter-process or inter-computer communcation between the client and the server, the issue of authentication is much simplıfied A misbehaving clients can bring down the server by exhaustıng resources but can not (modulo Java security) learn of other clients' data.

The ServerGui component: It is desirable to provide the abulity to remotely inspect the proactive network, or any specific node withın this network To achieve this, we created the ServerGui component. This component is not part of the Pserver, but rather an independent program whose purpose is to send requests to a Pserver and to display the server's responses. It is written as a Java applet and can be downloaded throught the browser The main request it supports is the "V1ew PServer's Status and information.

\section{References}

[1] H. Attiya, and J. Welch, Distributed Computing. fundamentals, simulations and advanced topics Mc.Grow-Hill, 1998

[2] G. R. Blakley, Safeguarding cryptographic keys. In Proc. AFIPS 1979 National Computer Conference, pp. 313-317. AFIPS, 1979.

[3] D. Boneh and M. Franklin. Efficient generation of shared RSA keys. In Proc. Crypto '97, pp. 425-539.

[4] R. Canetti, R. Gennaro, A. Herzberg and D. Naor, Proactive Security: Long-term protection against break-ins. CryptoBytes: the technical newsletter of RSA Labs, Vol. 3, number 1 - Spring, 1997.

[5] R. Canetti, S. Halevi, and A. Herzberg. "Maintaining authenticated communication in the presence of break-ins". To be published in Journal of Cryptography, 1999. An extended abstract of this paper appeared in the Proceedings of the 16th ACM Symp. on Principles of Distributed Computation. 1997. 
[6] R. Canetti and A. Herzberg. Maintaining security in the presence of transient faults In Crypto' 94, pp. 425-438, August, 1994.

17] CertCo, Root Authority, http://www.certco.com

[8] C.S. Chow and A. Herzberg. Network randomization protocol: A proactive pseudo-random generator. Appears in Proc. 5th USENIX UNIX Security Symposium, Salt Lake City, Utah, June 1995, pp. 55-63.

[9] P. Feldman. A Practical Scheme for non-Interactive verifiable secret sharing. In Proc.28th Annual Symp. on Foundations of Computer Science, pp. 427-437. IEEE, 1987.

[10] Y. Frankel, P. Gemmell, P. Mackenzie, and M. Yung. Optimal resilience proactive public-key cryptosystems. In Proc. 38th Annual Symp. on Foundations of Computer Science. IEEE, 1997.

[11] Y. Frankel, P. Gemmell, P. Mackenzie, and M. Yung. Proactive RSA. In Proc. of Crypto '97.

[12] P. Gemmell. An introduction to threshold cryptography. In Cryptobytes, Winter 97, pp. 7-12, 1997.

[13] R. Gennaro, S. Jarecki, H Krawczyk and T. Rabin, Robust threshold DSS signature. In Ueli Maurer, editor, Advances in Cryptology - Eurocrypt '96, pp. 354-371, 1996. Springer-Verlag Lecture Notes in Computer Science No. 1070.

[14] V. Hamilton, G. Istrail - Sandia National Labs. Implementation of proactive threshold public-key protocols, Proceedings of the 1998 RSA Data Security Conference.

[15] A. Herzberg, M. Jakobsson, S. Jarecki, H. Krawczyk and M. Yung. Proactive public key and signature systems, ACM Security' 97.

[16] A. Herzberg, S. Jarecki, H. Krawczyk, and M. Yung, Proactive secret sharing, or: How to cope with perpetual leakage. In D. Coopersmith, editor, Advances in Cryptology - Crypto '95, pp. 339-352, 1995. Lecture Notes in Computer Science No. 963.

[17] ITTC http://www.stanford.edu/-dabo/ITTC

[18] A. Iyengar, R. Cahn, C. Jutla and J.A. Garay, Design and implementation of a secure distributed data repository, in IFIP 1998.

[19] M. Malkin, T. Wu and D. Boneh, Experimenting with shared generation of RSA keys, in proceedings of the Internet Society's 1999 Symposium on Network and Distributed System Security (SNDSS), pp. 43-56.

[20] M. Naor, B. Pinkas and O. Reingold, Distributed pseudo-random functions and KDCs, to appear in Proc. of Eurocrypt ' 99.

[21] R. Ostrovsky and M. Yung, How to withstand mobile virus attacks, PODC 1991, pp.51-61.

[22] T. Pedersen. Non-interactive and information theoretic secure verifiable secret sharing. In D. Davies, editor,
Advances in Cryptology - Eurocrypto '91, pp. 522-526, 1991. Lecture Notes in Computer Science No. 547.

[23] T. Pedersen. A threshold cryptosystem without a trusted party in J. Feigenbaum, editor, Advances in Cryptology - Crypto '91, pp. 129-140, 1991. Lecture Notes in Computer Science No. 576

[24] T. Rabin, A simplified approach to threshold and proactive RSA, Proc. of Crypto ' 98.

[25] M. K. Reiter, The Rampart toolkit for bulding high-integrity services. In K. P. Birman, F. Mattern and A. Schiper, editors, Theory and Practice in Distributed Systems (LNCS 938), 99-110, Springer-Verlag, 1995.

[26] M. K. Reiter, Secure agreement protocols Reliable and atomic group multicast in Rampart Proc. 2nd ACM Conference on Computer and Communication Security, 1994.

[27] M. Reiter, M. Franklin, J. Lacy and R. Wright, The $\Omega$ Key Management Service, Proc. of the 3rd ACM Conference on Computer and Communication Security, 1996.

[28] A. Shamir. How to Share a Secret. Communications of the ACM, 22:612-613, 1979.

[29] T. Wu, M. Malkin and D. Boneh, Building intrusıon tolerant applications, submitted to 8th USENIX Security Symposium. 